\title{
Research Article \\ Existence of Periodic Solution for a Nonlinear Fractional Differential Equation
}

\author{
Mohammed Belmekki, ${ }^{1}$ Juan J. Nieto, ${ }^{2}$ \\ and Rosana Rodríguez-López ${ }^{2}$ \\ ${ }^{1}$ Département de Mathématiques, Université de Saïda, BP 138, 20000 Saïda, Algeria \\ ${ }^{2}$ Departamento de Análisis Matemático, Facultad de Matemáticas, Universidad de Santiago de Compostela, \\ 15782 Santiago de Compostela, Spain
}

Correspondence should be addressed to Rosana Rodríguez-López, rosana.rodriguez.lopez@usc.es

Received 2 February 2009; Revised 10 April 2009; Accepted 4 June 2009

Recommended by Donal O'Regan

We study the existence of solutions for a class of fractional differential equations. Due to the singularity of the possible solutions, we introduce a new and proper concept of periodic boundary value conditions. We present Green's function and give some existence results for the linear case and then we study the nonlinear problem.

Copyright (C) 2009 Mohammed Belmekki et al. This is an open access article distributed under the Creative Commons Attribution License, which permits unrestricted use, distribution, and reproduction in any medium, provided the original work is properly cited.

\section{Introduction}

Fractional calculus is a generalization of ordinary differentiation and integration to arbitrary noninteger order. The subject is as old as the differential calculus, and goes back to time when Leibnitz and Newton invented differential calculus. The idea of fractional calculus has been a subject of interest not only among mathematicians but also among physicists and engineers. See, for instance, [1-6].

Fractional-order models are more accurate than integer-order models, that is, there are more degrees of freedom in the fractional-order models. Furthermore, fractional derivatives provide an excellent instrument for the description of memory and hereditary properties of various materials and processes due to the existence of a "memory" term in a model. This memory term insures the history and its impact to the present and future. For more details, see [7].

Fractional calculus appears in rheology, viscoelasticity, electrochemistry, electromagnetism, and so forth. For details, see the monographs of Kilbas et al. [8], Kiryakova [9], Miller and Ross [10], Podlubny [11], Oldham and Spanier [12], and Samko et al. [13], and the papers of Diethelm et al. [14-16], Mainardi [17], Metzler et al. [18], Podlubny et al. [19], 
and the references therein. For some recent advances on fractional calculus and differential equations, see $[1,3,20-24]$. the form

In this paper we consider the following nonlinear fractional differential equation of

$$
D^{\delta} u(t)-\lambda u(t)=f(t, u(t)), \quad t \in J:=(0,1], 0<\delta<1,
$$

where $D^{\delta}$ is the standard Riemann-Liouville fractional derivative, $f$ is continuous, and $\lambda \in \mathbb{R}$.

This paper is organized as follows. in Section 2 we recall some definitions of fractional integral and derivative and related basic properties which will be used in the sequel. In Section 3, we deal with the linear case where $f(t, u(t))=\sigma(t)$ is a continuous function. Section 4 is devoted to the nonlinear case.

\section{Preliminary Results}

In this section, we introduce notations, definitions, and preliminary facts which are used throughout this paper.

Let $C[0,1]$ the Banach space of all continuous real functions defined on $[0,1]$ with the norm $\|f\|=: \sup \{|f(t)|: t \in[0,1]\}$. Define for $t \in[0,1], f_{r}(t)=t^{r} f(t)$. Let $C_{r}[0,1], r \geq 0$ be the space of all functions $f$ such that $f_{r} \in C[0,1]$ which turn out to be a Banach space when endowed with the norm $\|f\|_{r}=: \sup \left\{t^{r}|f(t)|: t \in[0,1]\right\}$.

By $L^{1}[0,1]$ we denote the space of all real functions defined on $[0,1]$ which are Lebesgue integrable.

Obviously $C_{r}[0,1] \subset L^{1}[0,1]$ if $r<1$.

Definition 2.1 (see $[11,13]$ ). The Riemann-Liouville fractional primitive of order $s>0$ of a function $f:(0,1] \rightarrow \mathbb{R}$ is given by

$$
I_{0}^{s} f(t)=\frac{1}{\Gamma(s)} \int_{0}^{t}(t-\tau)^{s-1} f(\tau) d \tau,
$$

provided the right side is pointwise defined on $(0,1]$, and where $\Gamma$ is the gamma function.

For instance, $I_{0}^{s}$ exists for all $s>0$, when $f \in C((0,1]) \cap L^{1}(0,1]$; note also that when $f \in C[0,1]$, then $I_{0}^{S} f \in C[0,1]$ and moreover $I_{0}^{S} f(0)=0$.

Let $0<s<1$, if $f \in C_{r}[0,1]$ with $r<s$, then $I^{s} f \in C[0,1]$, with $I^{s} f(0)=0$. If $f \in C_{s}[0,1]$, then $I^{s} f$ is bounded at the origin, whereas if $f \in C_{r}[0,1]$ with $s<r<1$, then we may expect $I^{s} f$ to be unbounded at the origin.

Recall that the law of composition $I^{r} I^{s}=I^{r+s}$ holds for all $r, s>0$.

Definition 2.2 (see $[11,13]$ ). The Riemann-Liouville fractional derivative of order $s>0$ of a continuous function $f:(0,1] \rightarrow \mathbb{R}$ is given by

$$
D^{s} f(t)=\frac{1}{\Gamma(1-s)} \frac{d}{d t} \int_{0}^{t}(t-\tau)^{-s} f(\tau) d \tau=\frac{d}{d t} I_{0}^{1-s} f(t) .
$$

We have $D^{s} I^{s} f=f$ for all $f \in C(0,1] \cap L^{1}(0,1]$. 
Lemma 2.3. Let $0<s<1$. If one assumes $u \in C(0,1] \cap L^{1}(0,1]$, then the fractional differential equation

$$
D^{s} u=0
$$

has $u(t)=c t^{s-1}, c \in \mathbb{R}$, as unique solutions.

From this lemma we deduce the following law of composition.

Proposition 2.4. Assume that $f$ is in $C(0,1] \cap L^{1}(0,1]$ with a fractional derivative of order $0<s<1$ that belongs to $C(0,1] \cap L^{1}(0,1]$. Then

$$
I^{s} D^{s} f(t)=f(t)+c t^{s-1}
$$

for some $c \in \mathbb{R}$.

$$
\text { If } f \in C_{r}[0,1] \text { with } r<1-s \text { and } D^{s} f \in C(0,1] \cap L^{1}(0,1] \text {, then } I^{s} D^{s} f=f \text {. }
$$

\section{Linear Problem}

In this section, we will be concerned with the following linear fractional differential equation:

$$
D^{\delta} u(t)-\lambda u(t)=\sigma(t), \quad t \in J:=(0,1], 0<\delta<1,
$$

where $\lambda \in \mathbb{R}$, and $\sigma$ is a continuous function.

Before stating our main results for this section, we study the equation

$$
D^{\delta} u(t)=\sigma(t), \quad t \in J:=(0,1], 0<\delta<1 .
$$

Then

$$
u(t)=c t^{\delta-1}+\left(I^{\delta} \sigma\right)(t), \quad t \in[0,1]
$$

for some $c \in \mathbb{R}$.

Note that $\left(I^{\delta} \sigma\right) \in W^{1,1}(0,1)$ and $\left(I^{\delta} \sigma\right)(0)=0$. However, $u \notin W^{1,1}(0,1)$ since $c t^{\delta-1}$ has a singularity at 0 for $c \neq 0$.

It is easy to show that $u \in C_{1-\delta}[0,1]$. Hence we should look for solutions, not in $W^{1,1}(0,1)$ but in $C_{1-\delta}[0,1]$. We cannot consider the usual initial condition $u(0)=u_{0}$, but $\lim _{t \mapsto 0^{+}} t^{1-\delta} u(t)=u_{0}$. Hence, to study the periodic boundary value problem, one has to consider the following boundary condition of periodic type

$$
\lim _{t \mapsto 0^{+}} t^{1-\delta} u(t)=u(1)
$$


From (3.3), we have

$$
\begin{gathered}
\lim _{t \mapsto 0^{+}} t^{1-\delta} u(t)=c, \\
u(1)=c+\left(I^{\delta} \sigma\right)(1)
\end{gathered}
$$

that leads to the following.

Theorem 3.1. The periodic boundary value problem (3.2)-(3.4) has a unique solution $u \in C_{1-\delta}[0,1]$ if and only if

$$
\int_{0}^{1} \frac{\sigma(s) d s}{(1-s)^{1-\delta}}=0
$$

The previous result remains true even if $\delta=1$. In this case, (3.2) is reduced to the ordinary differential equation

$$
u^{\prime}(t)=\sigma(t)
$$

with the periodic boundary condition

$$
u(0)=u(1)
$$

and the condition (3.6) is reduced to the classical one:

$$
\int_{0}^{1} \sigma(s) d s=0
$$

Now, for $\lambda$ different from 0 , consider the homogenous linear equation

$$
D^{\delta} u(t)-\lambda u(t)=0, \quad t \in J:=(0,1], 0<\delta<1 .
$$

The solution is given by

$$
u(t)=c \Gamma(\delta) \sum_{i=1}^{\infty} \frac{\lambda^{i-1}}{\Gamma(\delta i)} t^{\delta i-1}, \quad c \in \mathbb{R}
$$

Indeed, we have

$$
D^{\delta} u(t)=c \Gamma(\delta) \sum_{i=1}^{\infty} \frac{\lambda^{i-1}}{\Gamma(\delta i)} D^{\delta}\left(t^{\delta i-1}\right)
$$

since the series representing $u$ is absolutely convergent. 
Boundary Value Problems

Using the identities

$$
\begin{gathered}
D^{s} t^{\mu}=\frac{\Gamma(\mu+1)}{\Gamma(\mu+1-s)} t^{\mu-s}, \quad \mu>-1, \\
D^{s} t^{s-1}=0,
\end{gathered}
$$

we get

$$
D^{\delta}\left(t^{\delta i-1}\right)=\frac{\Gamma(\delta i)}{\Gamma(\delta(i-1))} t^{\delta(i-1)-1}, \quad \text { for } i>1, \quad D^{\delta} t^{\delta-1}=0
$$

Then

$$
\begin{aligned}
D^{\delta} u(t) & =c \Gamma(\delta) \sum_{i=2}^{\infty} \frac{\lambda^{i-1}}{\Gamma(\delta(i-1))} t^{\delta(i-1)-1} \\
& =\lambda c \Gamma(\delta) \sum_{i=2}^{\infty} \frac{\lambda^{i-2}}{\Gamma(\delta(i-1))} t^{\delta(i-1)-1} \\
& =\lambda c \Gamma(\delta) \sum_{i=1}^{\infty} \frac{\lambda^{i-1}}{\Gamma(\delta i)} t^{\delta i-1} \\
& =\lambda u(t) .
\end{aligned}
$$

Note that the solution can be expressed by means of the classical Mittag-Leffler special functions [8]. Indeed

$$
\begin{aligned}
u(t) & =c \Gamma(\delta) \sum_{i=1}^{\infty} \frac{\lambda^{i-1}}{\Gamma(\delta i)} t^{\delta i-1} \\
& =c \Gamma(\delta) t^{\delta-1} \sum_{i=1}^{\infty} \frac{\left(\lambda t^{\delta}\right)^{i-1}}{\Gamma(\delta i)} \\
& =c \Gamma(\delta) t^{\delta-1} \sum_{i=0}^{\infty} \frac{\left(\lambda t^{\delta}\right)^{i}}{\Gamma(\delta i+\delta)} \\
& =c \Gamma(\delta) t^{\delta-1} E_{\delta, \delta}\left(\lambda t^{\delta}\right) .
\end{aligned}
$$

The previous formula remains valid for $\delta=1$. In this case,

$$
\begin{gathered}
\Gamma(1)=1, \\
E_{1,1}(\lambda t)=E_{1}(\lambda t)=\exp (\lambda t) .
\end{gathered}
$$


Then

$$
u(t)=c \exp (\lambda t)
$$

which is the classical solution to the homogeneous linear differential equation

$$
u^{\prime}(t)-\lambda u(t)=0
$$

Now, consider the nonhomogeneous problem (3.1). We seek the particular solution in the following form:

$$
\begin{aligned}
u_{p}(t) & =\int_{0}^{t}(t-s)^{\delta-1} E_{\delta, \delta}\left(\lambda(t-s)^{\delta}\right) \sigma(s) d s \\
& =\int_{0}^{t}(t-s)^{\delta-1} \sum_{i=0}^{\infty} \frac{\lambda^{i}(t-s)^{i \delta}}{\Gamma(\delta(i+1))} \sigma(s) d s .
\end{aligned}
$$

It suffices to show that

$$
u_{p}(t)=\lambda\left(I^{\delta} u_{p}\right)(t)+\left(I^{\delta} \sigma\right)(t)
$$

Indeed

$$
\begin{aligned}
\left(I^{\delta} u_{p}\right)(t) & =\frac{1}{\Gamma(\delta)} \int_{0}^{t}(t-s)^{\delta-1} u_{p}(s) d s \\
& =\frac{1}{\Gamma(\delta)} \int_{0}^{t} \int_{0}^{s}(t-s)^{\delta-1}(s-\xi)^{\delta-1} \sum_{i=0}^{\infty} \frac{\lambda^{i}(s-\xi)^{i \delta}}{\Gamma(\delta(i+1))} \sigma(\xi) d \xi d s \\
& =\frac{1}{\Gamma(\delta)} \sum_{i=0}^{\infty} \frac{\lambda^{i}}{\Gamma(\delta(i+1))} \int_{0}^{t} \int_{0}^{s}(t-s)^{\delta-1}(s-\xi)^{\delta-1}(s-\xi)^{i \delta} \sigma(\xi) d \xi d s \\
& =\frac{1}{\Gamma(\delta)} \sum_{i=0}^{\infty} \frac{\lambda^{i}}{\Gamma(\delta(i+1))} \int_{0}^{t} \sigma(\xi) \int_{\xi}^{t}(t-s)^{\delta-1}(s-\xi)^{\delta(i+1)-1} d s d \xi
\end{aligned}
$$

Using the change of variable

$$
s=(1-\theta) \xi+\theta t
$$

we get

$$
\begin{aligned}
\left(I^{\delta} u_{p}\right)(t) & =\frac{1}{\Gamma(\delta)} \sum_{i=0}^{\infty} \frac{\lambda^{i}}{\Gamma(\delta(i+1))} \int_{0}^{t} \sigma(\xi) \int_{0}^{1}(1-\theta)^{\delta-1} \theta^{\delta(i+1)-1}(t-\xi)^{\delta(i+2)-1} d \theta d \xi \\
& =\frac{1}{\Gamma(\delta)} \sum_{i=0}^{\infty} \frac{\lambda^{i}}{\Gamma(\delta(i+1))} \int_{0}^{t} \frac{\Gamma(\delta(i+1)) \Gamma(\delta)}{\Gamma(\delta(i+2))}(t-\xi)^{\delta(i+2)-1} \sigma(\xi) d \xi .
\end{aligned}
$$


Then,

$$
\begin{aligned}
\lambda\left(I^{\delta} u_{p}\right)(t) & =\sum_{i=0}^{\infty} \frac{\lambda^{i+1}}{\Gamma(\delta(i+2))} \int_{0}^{t}(t-\xi)^{\delta-1}(t-\xi)^{\delta(i+1)} \sigma(\xi) d \xi \\
& =\int_{0}^{t}(t-\xi)^{\delta-1} \sum_{i=1}^{\infty} \frac{\lambda^{i}}{\Gamma(\delta(i+1))}(t-\xi)^{\delta i} \sigma(\xi) d \xi \\
& =\int_{0}^{t}(t-\xi)^{\delta-1}\left[\sum_{i=0}^{\infty} \frac{\lambda^{i}}{\Gamma(\delta(i+1))}(t-\xi)^{\delta i}-\frac{1}{\Gamma(\delta)}\right] \sigma(\xi) d \xi \\
& =\int_{0}^{t}(t-\xi)^{\delta-1} E_{\delta, \delta}\left(\lambda(t-\xi)^{\delta}\right) \sigma(\xi) d \xi-\frac{1}{\Gamma(\delta)} \int_{0}^{t}(t-\xi)^{\delta-1} \sigma(\xi) d \xi \\
& =u_{p}(t)-\left(I^{\delta} \sigma\right)(t) .
\end{aligned}
$$

Hence, the general solution of the nonhomogeneous equation (3.1) takes the form

$$
u(t)=c \Gamma(\delta) t^{\delta-1} E_{\delta, \delta}\left(\lambda t^{\delta}\right)+\int_{0}^{t}(t-s)^{\delta-1} E_{\delta, \delta}\left(\lambda(t-s)^{\delta}\right) \sigma(s) d s
$$

Now, consider the periodic boundary value problem (3.1)-(3.4). Its unique solution is given by (3.26) for some $c \in \mathbb{R}$. Also $u$ is in $C_{1-\delta}[0,1]$ and

$$
\lim _{t \mapsto 0^{+}} t^{1-\delta} u(t)=c
$$

From (3.26), we have

$$
u(1)=c \Gamma(\delta) E_{\delta, \delta}(\lambda)+\int_{0}^{1}(1-s)^{\delta-1} E_{\delta, \delta}\left(\lambda(1-s)^{\delta}\right) \sigma(s) d s
$$

which leads to

$$
c\left[1-\Gamma(\delta) E_{\delta, \delta}(\lambda)\right]=\int_{0}^{1}(1-s)^{\delta-1} E_{\delta, \delta}\left(\lambda(1-s)^{\delta}\right) \sigma(s) d s,
$$

since $\Gamma(\delta) E_{\delta, \delta}(\lambda) \neq 1$ for any $\lambda \neq 0$, we have

$$
c=\left[1-\Gamma(\delta) E_{\delta, \delta}(\lambda)\right]^{-1} \int_{0}^{1}(1-s)^{\delta-1} E_{\delta, \delta}\left(\lambda(1-s)^{\delta}\right) \sigma(s) d s .
$$


Then the solution of the problem (3.1)-(3.4) is given by

$$
\begin{aligned}
u(t)= & \frac{\Gamma(\delta)}{1-\Gamma(\delta) E_{\delta, \delta}(\lambda)} t^{\delta-1} E_{\delta, \delta}\left(\lambda t^{\delta}\right) \int_{0}^{1}(1-s)^{\delta-1} E_{\delta, \delta}\left(\lambda(1-s)^{\delta}\right) \sigma(s) d s \\
& +\int_{0}^{t}(t-s)^{\delta-1} E_{\delta, \delta}\left(\lambda(t-s)^{\delta}\right) \sigma(s) d s .
\end{aligned}
$$

Thus we have the following result.

Theorem 3.2. The periodic boundary value problem (3.1)-(3.4) has a unique solution $u \in C_{1-\delta}[0,1]$ given by

$$
u(t)=\int_{0}^{1} G_{\lambda, \delta}(t, s) \sigma(s) d s
$$

where

$$
\begin{aligned}
& G_{\lambda, \delta}(t, s) \\
& \quad= \begin{cases}\frac{\Gamma(\delta) E_{\delta, \delta}\left(\lambda t^{\delta}\right) E_{\delta, \delta}\left(\lambda(1-s)^{\delta}\right) t^{\delta-1}(1-s)^{\delta-1}}{1-\Gamma(\delta) E_{\delta, \delta}(\lambda)}+(t-s)^{\delta-1} E_{\delta, \delta}\left(\lambda(t-s)^{\delta}\right), & 0 \leq s \leq t \leq 1, \\
\frac{\Gamma(\delta) E_{\delta, \delta}\left(\lambda t^{\delta}\right) E_{\delta, \delta}\left(\lambda(1-s)^{\delta}\right) t^{\delta-1}(1-s)^{\delta-1}}{1-\Gamma(\delta) E_{\delta, \delta}(\lambda)}, & 0 \leq t<s \leq 1 .\end{cases}
\end{aligned}
$$

For $\lambda, \delta$ given, $G_{\lambda, \delta}$ is bounded on $[0,1] \times[0,1]$.

For $\delta=1,(3.1)$ is

$$
u^{\prime}(t)-\lambda u(t)=\sigma(t), \quad t \in J,
$$

and the boundary condition (3.4) is

$$
u(0)=u(1)
$$

In this situation Green's function is

$$
G_{\lambda, 1}(t, s)=\frac{1}{1-e^{\lambda}} \begin{cases}e^{\lambda(t-s)}, & 0 \leq s \leq t \leq 1, \\ e^{\lambda(1+t-s)}, & 0 \leq t<s \leq 1 .\end{cases}
$$

which is precisely Green's function for the periodic boundary value problem considered in $[25,26]$. 


\section{Nonlinear Problem}

In this section we will be concerned with the existence and uniqueness of solution to the nonlinear problem (1.1)-(3.4). To this end, we need the following fixed point theorem of Schaeffer.

Theorem 4.1. Assume $X$ to be a normed linear space, and let operator $F: X \rightarrow X$ be compact. Then either

(i) the operator $F$ has a fixed point in $X$, or

(ii) the set $\varepsilon=\{u \in X: u=\mu F(u), \mu \in(0,1)\}$ is unbounded.

If $u$ is a solution of problem (1.1)-(3.4), then it is given by

$$
u(t)=\int_{0}^{1} G_{l, \delta}(t, s) f(s, u(s)) d s,
$$

where $G_{\lambda, \delta}$ is Green's function defined in Theorem 3.2.

Define the operator $B: C_{1-\delta}[0,1] \rightarrow C_{1-\delta}[0,1]$ by

$$
B(u)(t)=\int_{0}^{1} G_{\lambda, \delta}(t, s) f(s, u(s)) d s, \quad t \in[0,1]
$$

Then the problem (1.1)-(3.4) has solutions if and only if the operator equation $B u=u$ has fixed points.

Lemma 4.2. Suppose that the following hold:

(i) there exists a constant $M>0$ such that

$$
|f(t, u)| \leq M, \quad \forall t \in[0,1], u \in \mathbb{R}
$$

(ii) there exists a constant $k>0$ such that

$$
|f(t, u)-f(t, v)| \leq k|u-v|, \quad \text { for each } t \in[0,1], \text { and all } u, v \in \mathbb{R} \text {. }
$$

Then the operator $B$ is well defined, continuous, and compact. 
Proof. (a) We check, using hypothesis (4.3), that $B u \in C_{1-\delta}[0,1]$, for every $u \in C_{1-\delta}[0,1]$. Indeed, for any $t_{1}<t_{2} \in[0,1], u \in D$, we have

$$
\begin{aligned}
& \left|t_{1}^{1-\delta} \boldsymbol{B}(u)\left(t_{1}\right)-t_{2}^{1-\delta} \boldsymbol{B}(u)\left(t_{2}\right)\right| \\
& =\left|t_{1}^{1-\delta} \int_{0}^{1} G_{\lambda, \delta}\left(t_{1}, s\right) f(s, u(s)) d s-t_{2}^{1-\delta} \int_{0}^{1} G_{\lambda, \delta}\left(t_{2}, s\right) f(s, u(s)) d s\right| \\
& \leq \mid \frac{\Gamma(\delta) E_{\delta, \delta}\left(\lambda t_{1}^{\delta}\right)}{1-\Gamma(\delta) E_{\delta, \delta}(\lambda)} \int_{0}^{t_{1}} E_{\delta, \delta}\left(\lambda(1-s)^{\delta}\right)(1-s)^{\delta-1} f(s, u(s)) d s \\
& +t_{1}^{1-\delta} \int_{0}^{t_{1}} E_{\delta, \delta}\left(\lambda\left(t_{1}-s\right)^{\delta}\right)\left(t_{1}-s\right)^{\delta-1} f(s, u(s)) d s \\
& -\frac{\Gamma(\delta) E_{\delta, \delta}\left(\lambda t_{2}^{\delta}\right)}{1-\Gamma(\delta) E_{\delta, \delta}(\lambda)} \int_{0}^{t_{1}} E_{\delta, \delta}\left(\lambda(1-s)^{\delta}\right)(1-s)^{\delta-1} f(s, u(s)) d s \\
& -t_{2}^{1-\delta} \int_{0}^{t_{1}} E_{\delta, \delta}\left(\lambda\left(t_{2}-s\right)^{\delta}\right)\left(t_{2}-s\right)^{\delta-1} f(s, u(s)) d s \\
& +\frac{\Gamma(\delta) E_{\delta, \delta}\left(\lambda t_{1}^{\delta}\right)}{1-\Gamma(\delta) E_{\delta, \delta}(\lambda)} \int_{t_{1}}^{t_{2}} E_{\delta, \delta}\left(\lambda(1-s)^{\delta}\right)(1-s)^{\delta-1} f(s, u(s)) d s \\
& -\frac{\Gamma(\delta) E_{\delta, \delta}\left(\lambda t_{2}^{\delta}\right)}{1-\Gamma(\delta) E_{\delta, \delta}(\lambda)} \int_{t_{1}}^{t_{2}} E_{\delta, \delta}\left(\lambda(1-s)^{\delta}\right)(1-s)^{\delta-1} f(s, u(s)) d s \\
& -t_{2}^{1-\delta} \int_{t_{1}}^{t_{2}} E_{\delta, \delta}\left(\lambda\left(t_{2}-s\right)^{\delta}\right)\left(t_{2}-s\right)^{\delta-1} f(s, u(s)) d s \\
& +\frac{\Gamma(\delta) E_{\delta, \delta}\left(\lambda t_{1}^{\delta}\right)}{1-\Gamma(\delta) E_{\delta, \delta}(\lambda)} \int_{t_{2}}^{1} E_{\delta, \delta}\left(\lambda(1-s)^{\delta}\right)(1-s)^{\delta-1} f(s, u(s)) d s \\
& -\frac{\Gamma(\delta) E_{\delta, \delta}\left(\lambda t_{2}^{\delta}\right)}{1-\Gamma(\delta) E_{\delta, \delta}(\lambda)} \int_{t_{2}}^{1} E_{\delta, \delta}\left(\lambda(1-s)^{\delta}\right)(1-s)^{\delta-1} f(s, u(s)) d s \mid \\
& \leq M\left(\frac{\Gamma(\delta)}{\left|1-\Gamma(\delta) E_{\delta, \delta}(\lambda)\right|}\left|E_{\delta, \delta}\left(\lambda t_{1}^{\delta}\right)-E_{\delta, \delta}\left(\lambda t_{2}^{\delta}\right)\right| \int_{0}^{t_{1}}\left|E_{\delta, \delta}\left(\lambda(1-s)^{\delta}\right)\right|(1-s)^{\delta-1} d s\right. \\
& +\int_{0}^{t_{1}}\left|t_{1}^{1-\delta}\left(t_{1}-s\right)^{\delta-1} E_{\delta, \delta}\left(\lambda\left(t_{1}-s\right)^{\delta}\right)-t_{2}^{1-\delta}\left(t_{2}-s\right)^{\delta-1} E_{\delta, \delta}\left(\lambda\left(t_{2}-s\right)^{\delta}\right)\right| d s \\
& +\frac{\Gamma(\delta)}{\left|1-\Gamma(\delta) E_{\delta, \delta}(\lambda)\right|}\left|E_{\delta, \delta}\left(\lambda t_{1}^{\delta}\right)-E_{\delta, \delta}\left(\lambda t_{2}^{\delta}\right)\right| \int_{t_{1}}^{t_{2}}\left|E_{\delta, \delta}\left(\lambda(1-s)^{\delta}\right)\right|(1-s)^{\delta-1} d s
\end{aligned}
$$




$$
\begin{aligned}
& +t_{2}^{1-\delta} \int_{t_{1}}^{t_{2}}\left|E_{\delta, \delta}\left(\lambda\left(t_{2}-s\right)^{\delta}\right)\right|\left(t_{2}-s\right)^{\delta-1} d s \\
& \left.+\frac{\Gamma(\delta)}{\left|1-\Gamma(\delta) E_{\delta, \delta}(\lambda)\right|}\left|E_{\delta, \delta}\left(\lambda t_{1}^{\delta}\right)-E_{\delta, \delta}\left(\lambda t_{2}^{\delta}\right)\right| \int_{t_{2}}^{1}\left|E_{\delta, \delta}\left(\lambda(1-s)^{\delta}\right)\right|(1-s)^{\delta-1} d s\right) .
\end{aligned}
$$

From the previous expression, we deduce that, if $\left|t_{1}-t_{2}\right| \rightarrow 0$, then

$$
\left|t_{1}^{1-\delta} \boldsymbol{B}(u)\left(t_{1}\right)-t_{2}^{1-\delta} \mathcal{B}(u)\left(t_{2}\right)\right| \longrightarrow 0
$$

Indeed, note that the integral $\int_{0}^{t_{1}}\left|E_{\delta, \delta}\left(\lambda(1-s)^{\delta}\right)\right|(1-s)^{\delta-1} d s$ is bounded by

$$
\sum_{j=0}^{\infty} \frac{|\lambda|^{j}}{\Gamma(\delta j+\delta)} \int_{0}^{t_{1}}(1-s)^{\delta-1} d s=\sum_{j=0}^{\infty} \frac{|\lambda|^{j}}{\Gamma(\delta j+\delta)} \frac{1}{\delta(j+1)}=E_{\delta, \delta+1}(|\lambda|) .
$$

A similar argument is useful to study the behavior of the last three terms of the long inequality above. On the other hand, if we denote by $H$ the second term in the right-hand side of that inequality, then it is satisfied that

$$
\begin{aligned}
H= & \int_{0}^{t_{1}}\left|t_{1}^{1-\delta} \sum_{j=0}^{\infty} \frac{\lambda^{j}\left(t_{1}-s\right)^{\delta j+\delta-1}}{\Gamma(\delta j+\delta)}-t_{2}^{1-\delta} \sum_{j=0}^{\infty} \frac{\lambda^{j}\left(t_{2}-s\right)^{\delta j+\delta-1}}{\Gamma(\delta j+\delta)}\right| d s \\
\leq & \int_{0}^{t_{1}}\left|t_{1}^{1-\delta}-t_{2}^{1-\delta}\right| \sum_{j=0}^{\infty} \frac{|\lambda|^{j}\left(t_{1}-s\right)^{\delta j+\delta-1}}{\Gamma(\delta j+\delta)} d s+\int_{0}^{t_{1}} t_{2}^{1-\delta} \sum_{j=0}^{\infty} \frac{|\lambda|^{j}\left|\left(t_{1}-s\right)^{\delta j+\delta-1}-\left(t_{2}-s\right)^{\delta j+\delta-1}\right|}{\Gamma(\delta j+\delta)} d s \\
\leq & \left|t_{1}^{1-\delta}-t_{2}^{1-\delta}\right| \sum_{j=0}^{\infty} \frac{|\lambda|^{j}}{\Gamma(\delta j+\delta)} \int_{0}^{t_{1}}\left(t_{1}-s\right)^{\delta j+\delta-1} d s \\
& +t_{2}^{1-\delta} \sum_{j=0}^{\infty} \frac{|\lambda|^{j}}{\Gamma(\delta j+\delta)} \int_{0}^{t_{1}}\left|\left(t_{1}-s\right)^{\delta j+\delta-1}-\left(t_{2}-s\right)^{\delta j+\delta-1}\right| d s .
\end{aligned}
$$

Note that

$$
\int_{0}^{t_{1}}\left(t_{1}-s\right)^{\delta j+\delta-1} d s=\frac{t_{1}^{\delta j+\delta}}{\delta j+\delta} \leq \frac{1}{\delta j+\delta}
$$

and, concerning $R_{j}=\int_{0}^{t_{1}}\left|\left(t_{1}-s\right)^{\delta j+\delta-1}-\left(t_{2}-s\right)^{\delta j+\delta-1}\right| d s$, we distinguish two cases. If $j$ is such that $\delta j+\delta-1 \geq 0$, then

$$
R_{j}=\int_{0}^{t_{1}}\left(\left(t_{2}-s\right)^{\delta j+\delta-1}-\left(t_{1}-s\right)^{\delta j+\delta-1}\right) d s=\frac{-\left(t_{2}-t_{1}\right)^{\delta j+\delta}+t_{2}^{\delta j+\delta}-t_{1}^{\delta j+\delta}}{\delta j+\delta},
$$


and, if $j$ is such that $\delta j+\delta-1 \leq 0$, then

$$
R_{j}=\int_{0}^{t_{1}}\left(\left(t_{1}-s\right)^{\delta j+\delta-1}-\left(t_{2}-s\right)^{\delta j+\delta-1}\right) d s=\frac{\left(t_{2}-t_{1}\right)^{\delta j+\delta}+t_{1}^{\delta j+\delta}-t_{2}^{\delta j+\delta}}{\delta j+\delta} .
$$

In consequence,

$$
\begin{aligned}
H & \leq\left|t_{1}^{1-\delta}-t_{2}^{1-\delta}\right| \sum_{j=0}^{\infty} \frac{|\lambda|^{j}}{\Gamma(\delta j+\delta)(\delta j+\delta)}+t_{2}^{1-\delta} \sum_{j=0}^{\infty} \frac{|\lambda|^{j}}{\Gamma(\delta j+\delta)(\delta j+\delta)} R_{j} \\
& =\left|t_{1}^{1-\delta}-t_{2}^{1-\delta}\right| E_{\delta, \delta+1}(|\lambda|)+t_{2}^{1-\delta} \sum_{j=0}^{\infty} \frac{|\lambda|^{j}}{\Gamma(\delta j+\delta+1)} R_{j} .
\end{aligned}
$$

The first term in the right-hand side of the previous inequality clearly tends to zero as $\mid t_{1}-$ $t_{2} \mid \rightarrow 0$. On the other hand, denoting by [.] the integer part function, we have

$$
\begin{aligned}
\sum_{j=0}^{\infty} \frac{|\lambda|^{j}}{\Gamma(\delta j+\delta+1)} R_{j}= & \sum_{j=0}^{[1 / \delta]-1} \frac{|\lambda|^{j}}{\Gamma(\delta j+\delta+1)}\left(\left(t_{2}-t_{1}\right)^{\delta j+\delta}+t_{1}^{\delta j+\delta}-t_{2}^{\delta j+\delta}\right) \\
& +\sum_{j=[1 / \delta]}^{+\infty} \frac{|\lambda|^{j}}{\Gamma(\delta j+\delta+1)}\left(t_{2}^{\delta j+\delta}-t_{1}^{\delta j+\delta}-\left(t_{2}-t_{1}\right)^{\delta j+\delta}\right) .
\end{aligned}
$$

The finite sum obviously has limit zero as $\left|t_{1}-t_{2}\right| \rightarrow 0$. The infinite sum is equal to

$$
t_{2}^{\delta} \sum_{j=[1 / \delta]}^{+\infty} \frac{\left(|\lambda| t_{2}^{\delta}\right)^{j}}{\Gamma(\delta j+\delta+1)}-t_{1}^{\delta} \sum_{j=[1 / \delta]}^{+\infty} \frac{\left(|\lambda| t_{1}^{\delta}\right)^{j}}{\Gamma(\delta j+\delta+1)}-\left(t_{2}-t_{1}\right)^{\delta} \sum_{j=[1 / \delta]}^{+\infty} \frac{\left(|\lambda|\left(t_{2}-t_{1}\right)^{\delta}\right)^{j}}{\Gamma(\delta j+\delta+1)}
$$

and its limit as $\left|t_{1}-t_{2}\right| \rightarrow 0$ is zero. Note that $\sum_{j=[1 / \delta]}^{+\infty}\left(\left(|\lambda|\left(t_{2}-t_{1}\right)^{\delta}\right)^{j} / \Gamma(\delta j+\delta+1)\right)$ is bounded above by $\sum_{j=0}^{+\infty}\left(|\lambda|^{j} / \Gamma(\delta j+\delta+1)\right)=E_{\delta, \delta+1}(|\lambda|)$.

The previous calculus shows that $B u \in C_{1-\delta}[0,1]$, for $u \in C_{1-\delta}[0,1]$, hence we can define $B: C_{1-\delta}[0,1] \rightarrow C_{1-\delta}[0,1]$.

(b) Next, we prove that $B$ is continuous.

Note that, for $u, v \in C_{1-\delta}[0,1]$ and for every $t \in[0,1]$, we have, using hypothesis (4.4),

$$
\begin{aligned}
t^{1-\delta}|\boldsymbol{B}(u)(t)-\boldsymbol{B}(v)(t)| & \leq t^{1-\delta} \int_{0}^{1}\left|G_{\lambda, \delta}(t, s)\right||f(s, u(s))-f(s, v(s))| d s \\
& \leq k t^{1-\delta} \int_{0}^{1}\left|G_{\lambda, \delta}(t, s) \| u(s)-v(s)\right| d s .
\end{aligned}
$$


Using the definition of $\|\cdot\|_{1-\delta}$, we get

$$
\|\boldsymbol{B}(u)-\boldsymbol{B}(v)\|_{1-\delta} \leq k \max _{t \in[0,1]}\left\{t^{1-\delta} \int_{0}^{1}\left|G_{l, \delta}(t, s)\right| s^{\delta-1} d s\right\}\|u-v\|_{1-\delta} .
$$

Moreover,

$$
\begin{aligned}
& t^{1-\delta} \int_{0}^{1}\left|G_{\lambda, \delta}(t, s)\right| s^{\delta-1} d s=\int_{0}^{t} \frac{\Gamma(\delta)\left|E_{\delta, \delta}\left(\lambda t^{\delta}\right)\right|\left|E_{\delta, \delta} \lambda(1-s)^{\delta}\right|}{\left|1-\Gamma(\delta) E_{\delta, \delta}(\lambda)\right|}(1-s)^{\delta-1} s^{\delta-1} d s \\
& +t^{1-\delta} \int_{0}^{t}(t-s)^{\delta-1}\left|E_{\delta, \delta}\left(\lambda(t-s)^{\delta}\right)\right| s^{\delta-1} d s \\
& +\int_{t}^{1 \Gamma(\delta)\left|E_{\delta, \delta}\left(\lambda t^{\delta}\right)\right|\left|E_{\delta, \delta}\left(\lambda(1-s)^{\delta}\right)\right|}(1-s)^{\delta-1} s^{\delta-1} d s \\
& \leq \frac{\Gamma(\delta)\left|E_{\delta, \delta}\left(\lambda t^{\delta}\right)\right|}{\left|1-\Gamma(\delta) E_{\delta, \delta}(\lambda)\right|} \sum_{j=0}^{\infty} \frac{|\lambda|^{j}}{\Gamma(\delta j+\delta)} \int_{0}^{1}(1-s)^{\delta j+\delta-1} s^{\delta-1} d s \\
& +t^{1-\delta} \sum_{j=0}^{\infty} \frac{|\lambda|^{j}}{\Gamma(\delta j+\delta)} \int_{0}^{t}(t-s)^{\delta j+\delta-1} s^{\delta-1} d s .
\end{aligned}
$$

Using that $0 \leq 1-s \leq 1$ for $s \in[0,1], 0 \leq t-s \leq 1$ for $s \in[0, t]$, and $\delta j \geq 0$ for $j=0,1, \ldots$, we obtain

$$
\begin{aligned}
t^{1-\delta} \int_{0}^{1}\left|G_{\lambda, \delta}(t, s)\right| s^{\delta-1} d s \\
\leq \frac{\Gamma(\delta)\left|E_{\delta, \delta}\left(\lambda t^{\delta}\right)\right|}{\left|1-\Gamma(\delta) E_{\delta, \delta}(\lambda)\right|} \sum_{j=0}^{\infty} \frac{|\lambda|^{j}}{\Gamma(\delta j+\delta)} \int_{0}^{1}(1-s)^{\delta-1} s^{\delta-1} d s \\
\quad+t^{1-\delta} \sum_{j=0}^{\infty} \frac{|\lambda|^{j}}{\Gamma(\delta j+\delta)} \int_{0}^{t}(t-s)^{\delta-1} s^{\delta-1} d s \\
\leq \frac{\Gamma(\delta)\left|E_{\delta, \delta}\left(\lambda t^{\delta}\right)\right|}{\left|1-\Gamma(\delta) E_{\delta, \delta}(\lambda)\right|} E_{\delta, \delta}(|\lambda|) \int_{0}^{1}(1-s)^{\delta-1} s^{\delta-1} d s \\
\quad+t^{1-\delta} E_{\delta, \delta}(|\lambda|) \int_{0}^{t}(t-s)^{\delta-1} s^{\delta-1} d s .
\end{aligned}
$$

Note that the Beta function, also called the Euler integral of the first kind,

$$
\beta(p, q)=\int_{0}^{1} x^{p-1}(1-x)^{q-1} d x
$$


where $p>0$ and $q>0$, satisfies that $\beta(p, q)=\Gamma(p) \Gamma(q) / \Gamma(p+q)$. In particular, $\beta(\delta, \delta)=$ $(\Gamma(\delta))^{2} / \Gamma(2 \delta)$. On the other hand, using the change of variable $s=u t /(1+u)$, we deduce that

$$
\begin{aligned}
\int_{0}^{t} s^{p-1}(t-s)^{q-1} d s & =\int_{0}^{+\infty}\left(\frac{u t}{1+u}\right)^{p-1}\left(\frac{t}{1+u}\right)^{q-1}\left(\frac{t}{(1+u)^{2}}\right) d u \\
& =t^{p+q-1} \int_{0}^{+\infty} \frac{u^{p-1}}{(1+u)^{p+q}} d u=t^{p+q-1} \beta(p, q)=t^{p+q-1} \frac{\Gamma(p) \Gamma(q)}{\Gamma(p+q)}
\end{aligned}
$$

This proves that

$$
\int_{0}^{t} s^{\delta-1}(t-s)^{\delta-1} d s=t^{2 \delta-1} \frac{(\Gamma(\delta))^{2}}{\Gamma(2 \delta)}
$$

Hence,

$$
\begin{aligned}
& t^{1-\delta} \int_{0}^{1}\left|G_{\lambda, \delta}(t, s)\right| s^{\delta-1} d s \\
& \quad \leq \frac{\Gamma(\delta)\left|E_{\delta, \delta}\left(\lambda t^{\delta}\right)\right|}{\left|1-\Gamma(\delta) E_{\delta, \delta}(\lambda)\right|} E_{\delta, \delta}(|\lambda|) \frac{(\Gamma(\delta))^{2}}{\Gamma(2 \delta)}+t^{1-\delta} E_{\delta, \delta}(|\lambda|) t^{2 \delta-1} \frac{(\Gamma(\delta))^{2}}{\Gamma(2 \delta)} \\
& \quad=\left(\frac{\Gamma(\delta)\left|E_{\delta, \delta}\left(\lambda t^{\delta}\right)\right|}{\left|1-\Gamma(\delta) E_{\delta, \delta}(\lambda)\right|}+t^{\delta}\right) E_{\delta, \delta}(|\lambda|) \frac{(\Gamma(\delta))^{2}}{\Gamma(2 \delta)} \\
& \quad \leq\left(\frac{\Gamma(\delta) E_{\delta, \delta}(|\lambda|)}{\left|1-\Gamma(\delta) E_{\delta, \delta}(\lambda)\right|}+1\right) E_{\delta, \delta}(|\lambda|) \frac{(\Gamma(\delta))^{2}}{\Gamma(2 \delta)}
\end{aligned}
$$

In consequence,

$$
\|\mathbb{B}(u)-\mathcal{B}(v)\|_{1-\delta} \leq k\left(\frac{\Gamma(\delta) E_{\delta, \delta}(|\lambda|)}{\left|1-\Gamma(\delta) E_{\delta, \delta}(\lambda)\right|}+1\right) E_{\delta, \delta}(|\lambda|) \frac{(\Gamma(\delta))^{2}}{\Gamma(2 \delta)}\|u-v\|_{1-\delta}
$$

Finally, we check that $B$ is compact. Let $D$ be a bounded set in $C_{1-\delta}[0,1]$.

(i) First, we check that $\left\{t^{1-\delta}[B u](t): u \in D\right\}$ is a bounded set in $C[0,1]$. Indeed,

$$
\begin{aligned}
& t^{1-\delta} \int_{0}^{1}\left|G_{\lambda, \delta}(t, s)\right| d s \\
& \quad \leq \int_{0}^{t} \frac{\Gamma(\delta)\left|E_{\delta, \delta}\left(\lambda t^{\delta}\right) E_{\delta, \delta}\left(\lambda(1-s)^{\delta}\right)\right|(1-s)^{\delta-1}}{\left|1-\Gamma(\delta) E_{\delta, \delta}(\lambda)\right|} d s \\
& \quad+t^{1-\delta} \int_{0}^{t}(t-s)^{\delta-1}\left|E_{\delta, \delta}\left(\lambda(t-s)^{\delta}\right)\right| d s
\end{aligned}
$$


Boundary Value Problems

$$
\begin{aligned}
& +\int_{t}^{1 \Gamma(\delta)\left|E_{\delta, \delta}\left(\lambda t^{\delta}\right) E_{\delta, \delta}\left(\lambda(1-s)^{\delta}\right)\right|(1-s)^{\delta-1}} \frac{\left|1-\Gamma(\delta) E_{\delta, \delta}(\lambda)\right|}{\mid 1} d s \\
& =\frac{\Gamma(\delta)\left|E_{\delta, \delta}\left(\lambda t^{\delta}\right)\right|}{\left|1-\Gamma(\delta) E_{\delta, \delta}(\lambda)\right|} \int_{0}^{1}\left|E_{\delta, \delta}\left(\lambda(1-s)^{\delta}\right)\right|(1-s)^{\delta-1} d s \\
& +t^{1-\delta} \int_{0}^{t}(t-s)^{\delta-1}\left|E_{\delta, \delta}\left(\lambda(t-s)^{\delta}\right)\right| d s \\
& \leq \frac{\Gamma(\delta)\left|E_{\delta, \delta}\left(\lambda t^{\delta}\right)\right|}{\left|1-\Gamma(\delta) E_{\delta, \delta}(\lambda)\right|} \int_{0}^{1} \sum_{j=0}^{\infty} \frac{\left(|\lambda|(1-s)^{\delta}\right)^{j}}{\Gamma(\delta j+\delta)}(1-s)^{\delta-1} d s \\
& +t^{1-\delta} \int_{0}^{t} \sum_{j=0}^{\infty} \frac{\left(|\lambda|(t-s)^{\delta}\right)^{j}}{\Gamma(\delta j+\delta)}(t-s)^{\delta-1} d s \\
& =\frac{\Gamma(\delta)\left|E_{\delta, \delta}\left(\lambda t^{\delta}\right)\right|}{\left|1-\Gamma(\delta) E_{\delta, \delta}(\lambda)\right|} \sum_{j=0}^{\infty} \frac{|\lambda|^{j}}{\Gamma(\delta j+\delta)} \int_{0}^{1}(1-s)^{\delta(j+1)-1} d s \\
& +t^{1-\delta} \sum_{j=0}^{\infty} \frac{|\lambda|^{j}}{\Gamma(\delta j+\delta)} \int_{0}^{t}(t-s)^{\delta(j+1)-1} d s \\
& =\frac{\Gamma(\delta)\left|E_{\delta, \delta}\left(\lambda t^{\delta}\right)\right|}{\left|1-\Gamma(\delta) E_{\delta, \delta}(\lambda)\right|} \sum_{j=0}^{\infty} \frac{|\lambda|^{j}}{\Gamma(\delta j+\delta)} \frac{1}{\delta(j+1)}+t^{1-\delta} \sum_{j=0}^{\infty} \frac{|\lambda|^{j}}{\Gamma(\delta j+\delta)} \frac{t^{\delta(j+1)}}{\delta j+\delta} \\
& =\frac{\Gamma(\delta)\left|E_{\delta, \delta}\left(\lambda t^{\delta}\right)\right|}{\left|1-\Gamma(\delta) E_{\delta, \delta}(\lambda)\right|} \sum_{j=0}^{\infty} \frac{|\lambda|^{j}}{\Gamma(\delta j+\delta+1)}+t \sum_{j=0}^{\infty} \frac{|\lambda|^{j} t^{\delta j}}{\Gamma(\delta j+\delta+1)} \\
& =\frac{\Gamma(\delta)\left|E_{\delta, \delta}\left(\lambda t^{\delta}\right)\right|}{\left|1-\Gamma(\delta) E_{\delta, \delta}(\lambda)\right|} E_{\delta, \delta+1}(|\lambda|)+t E_{\delta, \delta+1}\left(|\lambda| t^{\delta}\right) .
\end{aligned}
$$

Hence

$$
t^{1-\delta}|\boldsymbol{B}(u)(t)| \leq M t^{1-\delta} \int_{0}^{1}\left|G_{\lambda, \delta}(t, s)\right| d s \leq M\left(\frac{\Gamma(\delta)\left|E_{\delta, \delta}\left(\lambda t^{\delta}\right)\right|}{\left|1-\Gamma(\delta) E_{\delta, \delta}(\lambda)\right|}+1\right) E_{\delta, \delta+1}(|\lambda|),
$$

and then

$$
\|\mathbb{B}(u)\|_{1-\delta} \leq M\left(\frac{\Gamma(\delta) E_{\delta, \delta}(|\lambda|)}{\left|1-\Gamma(\delta) E_{\delta, \delta}(\lambda)\right|}+1\right) E_{\delta, \delta+1}(|\lambda|)<\infty
$$

which implies that $\left\{t^{1-\delta}[B u](t): u \in D\right\}$ is a bounded set in $C[0,1]$.

(ii) Now, we prove that $\left\{t^{1-\delta}[B u](t): u \in D\right\}$ is an equicontinuous set in $C[0,1]$. Following the calculus in (a), we show that $\left|t_{1}^{1-\delta} \mathcal{B}(u)\left(t_{1}\right)-t_{2}^{1-\delta} \mathcal{B}(u)\left(t_{2}\right)\right|$ tends to zero as $t_{1} \mapsto t_{2}$.

Then $\left\{[B u]_{1-\delta}: u \in D\right\}$ is equicontinuous in the space $C[0,1]$, where $v_{1-\delta}(t)=t^{1-\delta} v(t)$, for $v \in C_{1-\delta}[0,1]$. 
As a consequence of (i) and (ii), $\left\{[B u]_{1-\delta}: u \in D\right\}$ is a bounded and equicontinuous set in the space $C[0,1]$.

Hence, for a sequence $\left\{u_{n}\right\}$ in $D,\left\{\left[B u_{n}\right]_{1-\delta}: n \in \mathbb{N}\right\}=\left\{t^{1-\delta}\left[\mathbb{B} u_{n}\right](t): n \in \mathbb{N}\right\}$ has a subsequence converging to $\varphi \in C[0, T]$, that is,

$$
\lim _{n \rightarrow \infty}\left|t^{1-\delta}\left[ß u_{n_{k}}\right](t)-\varphi(t)\right|=0, \quad \text { uniformly on } t \in[0,1]
$$

Taking $\psi(t)=t^{\delta-1} \varphi(t)$, we get

$$
\lim _{n \rightarrow \infty}\left|t^{1-\delta}\left[B u_{n_{k}}\right](t)-t^{1-\delta} \psi(t)\right|=0, \quad \text { uniformly on } t \in[0,1]
$$

which means that $\left\{B u_{n_{k}}\right\} \stackrel{C_{1-\delta}[0,1]}{\longrightarrow} \psi$, which proves that $B$ is compact.

Theorem 4.3. Assume that (4.3) and (4.4) hold. Then the problem (1.1)-(3.4) has at least one solution in $C_{1-\delta}[0,1]$.

Proof. Consider the set $\varepsilon=\{u \in X: u=\mu ß(u), \mu \in(0,1)\}$. we have

Let $u$ be any element of $\mathcal{\varepsilon}$, then $u=\mu 乃(u)$ for some $\mu \in(0,1)$. Thus for each $t \in[0,1]$,

$$
|u(t)| \leq \mu \int_{0}^{1}\left|G_{\lambda, \delta}(t, s)\right||f(s, u(s))| d s .
$$

As in Lemma 4.2, (i), we have

$$
\|u\|_{1-\delta} \leq M\left(\frac{\Gamma(\delta) E_{\delta, \delta}(|\lambda|)}{\left|1-\Gamma(\delta) E_{\delta, \delta}(\lambda)\right|}+1\right) E_{\delta, \delta+1}(|\lambda|)<\infty
$$

which implies that the set $\varepsilon$ is bounded independently of $\mu \in(0,1)$. Using Lemma 4.2 and Theorem 4.1, we obtain that the operator $B$ has at least a fixed point.

Remark 4.4. In Lemma 4.2, condition (4.3) is used to prove that the operator $B$ is continuous. Hence, in Lemma 4.2 and, in consequence, in Theorem 4.3, we can assume the weaker condition.

(i) For each $u_{0} \in C_{1-\delta}[0,1]$ fixed, there exists $k_{u_{0}}>0$ such that

$$
\left|f(t, u)-f\left(t, u_{0}(t)\right)\right| \leq k_{u_{0}}\left|u-u_{0}(t)\right|, \quad \text { for each } t \in[0,1], \text { and all } u \in \mathbb{R},
$$

instead of (4.3).

However, to prove the existence and uniqueness of solution given in the following theorem, we need to assume the Lipschitzian character of $f$ (condition (4.3)). 
Theorem 4.5. Assume that (4.4) holds. Then the problem (1.1)-(3.4) has a unique solution in $C_{1-\delta}[0,1]$, provided that

$$
k \frac{(\Gamma(\delta))^{2}}{\Gamma(2 \delta)} E_{\delta, \delta}(|\lambda|)\left(\frac{\Gamma(\delta) E_{\delta, \delta}(|\lambda|)}{\left|1-\Gamma(\delta) E_{\delta, \delta}(\lambda)\right|}+1\right)<1 .
$$

Proof. We use the Banach contraction principle to prove that the operator $\boldsymbol{B}$ has a unique fixed point.

Using the calculus in (b) Lemma 4.2, $B$ is a contraction by condition (4.32). As a consequence of Banach fixed point theorem, we deduce that $B$ has a unique fixed point which gives rise to a unique solution of problem (1.1)-(3.4).

Remark 4.6. If $\delta=1$, condition (4.32) is reduced to

$$
k e^{|\lambda|}\left(\frac{e^{|\lambda|}}{\left|1-e^{\lambda}\right|}+1\right)<1
$$

\section{Acknowledgment}

The research of J. J. Nieto and R. Rodríguez-López has been partially supported by Ministerio de Educacion y Ciencia and FEDER, project MTM2007-61724, and by Xunta de Galicia and FEDER, project PGIDIT06PXIB207023PR.

\section{References}

[1] B. Ahmad and J. J. Nieto, "Existence results for a coupled system of nonlinear fractional differential equations with three-point boundary conditions," preprint.

[2] B. Ahmad and J. J. Nieto, "Existence results for nonlinear boundary value problems of fractional integrodifferential equations with integral boundary conditions," Boundary Value Problems, vol. 2009, Article ID 708576, 11 pages, 2009.

[3] M. Benchohra, A. Cabada, and D. Seba, "An existence result for nonlinear fractional differential equations on Banach spaces," Boundary Value Problems, vol. 2009, Article ID 628916, 11 pages, 2009.

[4] B. Bonilla, M. Rivero, L. Rodríguez-Germá, and J. J. Trujillo, "Fractional differential equations as alternative models to nonlinear differential equations," Applied Mathematics and Computation, vol. 187, no. 1, pp. 79-88, 2007.

[5] V. Daftardar-Gejji and S. Bhalekar, "Boundary value problems for multi-term fractional differential equations," Journal of Mathematical Analysis and Applications, vol. 345, no. 2, pp. 754-765, 2008.

[6] V. Varlamov, "Differential and integral relations involving fractional derivatives of Airy functions and applications," Journal of Mathematical Analysis and Applications, vol. 348, no. 1, pp. 101-115, 2008.

[7] M. P. Lazarević and A. M. Spasić, "Finite-time stability analysis of fractional order time-delay systems: Gronwall's approach," Mathematical and Computer Modelling, vol. 49, no. 3-4, pp. 475-481, 2009.

[8] A. A. Kilbas, H. M. Srivastava, and J. J. Trujillo, Theory and Applications of Fractional Differential Equations, vol. 204 of North-Holland Mathematics Studies, Elsevier Science B.V., Amsterdam, The Netherlands, 2006.

[9] V. Kiryakova, Generalized Fractional Calculus and Applications, vol. 301 of Pitman Research Notes in Mathematics Series, Longman Scientific \& Technical, Harlow, UK, 1994.

[10] K. S. Miller and B. Ross, An Introduction to the Fractional Calculus and Fractional Differential Equations, A Wiley-Interscience Publication, John Wiley \& Sons, New York, NY, USA, 1993.

[11] I. Podlubny, Fractional Differential Equations, vol. 198 of Mathematics in Science and Engineering, Academic Press, San Diego, Calif, USA, 1999.

[12] K. B. Oldham and J. Spanier, The Fractional Calculus, Academic Press, New York, NY, USA, 1974. 
[13] S. G. Samko, A. A. Kilbas, and O. I. Marichev, Fractional Integrals and Derivatives. Theory and Applications, Gordon and Breach Science, Yverdon, Switzerland, 1993.

[14] K. Diethelm and A. D. Freed, "On the solution of nonlinear fractional order differential equations used in the modeling of viscoplasticity," in Scientifice Computing in Chemical Engineering II-Computational Fluid Dynamics, Reaction Engineering and Molecular Properties, F. Keil, W. Mackens, H. Voss, and J. Werther, Eds., pp. 217-224, Springer, Heidelberg, Germany, 1999.

[15] K. Diethelm and N. J. Ford, "Analysis of fractional differential equations," Journal of Mathematical Analysis and Applications, vol. 265, no. 2, pp. 229-248, 2002.

[16] K. Diethelm and G. Walz, "Numerical solution of fractional order differential equations by extrapolation," Numerical Algorithms, vol. 16, no. 3-4, pp. 231-253, 1997.

[17] F. Mainardi, "Fractional calculus: some basic problems in continuum and statistical mechanis," in Fractals and Fractional Calculus in Continuum Mechanics, A. Carpinteri and F. Mainardi, Eds., pp. 291348, Springer, Wien, Austria, 1997.

[18] R. Metzler, W. Schick, H.-G. Kilian, and T. F. Nonnenmacher, "Relaxation in filled polymers: a fractional calculus approach," The Journal of Chemical Physics, vol. 103, no. 16, pp. 7180-7186, 1995.

[19] I. Podlubny, I. Petráš, B. M. Vinagre, P. O’Leary, and L. Dorčák, "Analogue realizations of fractionalorder controllers," Nonlinear Dynamics, vol. 29, no. 1-4, pp. 281-296, 2002.

[20] D. Araya and C. Lizama, "Almost automorphic mild solutions to fractional differential equations," Nonlinear Analysis: Theory, Methods E Applications, vol. 69, no. 11, pp. 3692-3705, 2008.

[21] M. Benchohra, S. Hamani, J. J. Nieto, and B. A. Slimani, "Existence results for fractional differential inclusions with fractional order and impulses," to appear in Computers $\mathcal{E}$ Mathematics with Applications.

[22] Y.-K. Chang and J. J. Nieto, "Some new existence results for fractional differential inclusions with boundary conditions," Mathematical and Computer Modelling, vol. 49, no. 3-4, pp. 605-609, 2009.

[23] Y.-K. Chang and J. J. Nieto, "Existence of solutions for impulsive neutral integro-differential inclusions with nonlocal initial conditions via fractional operators," Numerical Functional Analysis and Optimization, vol. 30, pp. 227-244, 2009.

[24] H. Jafari and S. Seifi, "Homotopy analysis method for solving linear and nonlinear fractional diffusion-wave equation," Communications in Nonlinear Science and Numerical Simulation, vol. 14, no. 5, pp. 2006-2012, 2009.

[25] J. Li, J. J. Nieto, and J. Shen, "Impulsive periodic boundary value problems of first-order differential equations," Journal of Mathematical Analysis and Applications, vol. 325, no. 1, pp. 226-236, 2007.

[26] J. J. Nieto, "Differential inequalities for functional perturbations of first-order ordinary differential equations," Applied Mathematics Letters, vol. 15, no. 2, pp. 173-179, 2002. 